\section{Influence of ethnic origin on the incidence of keratoconus and associated atopic disease in Asians and white patients}

T Georgiou, CL Funnell, A Cassels-Brown and $\mathrm{R} \mathrm{O}^{\prime} \mathrm{Conor}$
Eye (2004) 18, 379-383. doi:10.1038/

sj.eye. 6700652

Keywords: keratoconus; atopy; Asian; Caucasian; incidence

\section{Introduction}

Keratoconus is a relatively common corneal ectasia causing significant visual disability. It is characterized by progressive irregular myopic astigmatism, central corneal thinning, and protrusion. ${ }^{1}$ Although the natural history of the disease has been well described, the aetiology and underlying biochemical pathology remains unconfirmed. Hereditary as well as environmental factors have been implicated. Working in a district hospital with a mixed ethnic population, it became evident that the majority of patients with keratoconus attending clinic were of Asian origin. There appeared to be an ethnic factor involved in the disease in this population.

\section{Materials and methods}

Retrospective study of new patients referred to Dewsbury District General Hospital, in

Yorkshire, and diagnosed with keratoconus in a 6-year period between July 1994 and July 2000.

The case notes of all patients newly diagnosed with keratoconus, as identified by the hospital coding system, were reviewed. Since 1995, all patients attending Dewsbury hospital have been coded, via the GP letter and entered into the hospital database. Coding is checked at each follow-up visit. The coding system should have identified most, if not all, patients presenting with keratoconus. There is no logical bias for under-coding of Asian or white patients.
Dewsbury District Hospital Yorkshire, UK

Correspondence:

CL Funnell

C/o Ophthalmic Secretaries

Clarendon Wing

Leeds General Infirmary

St George Street, Leeds

Yorkshire LS1 3EX, UK

Tel: + 441132432799

Fax: +44 1132926239

E-mail: c.l.funnell@

doctors.org.uk

Received: 10 September 2002

Accepted in revised form: 1 May 2003

Presented as a Poster at the Annual Congress Manchester 2002 
The patients in the study all came from North Kirklees catchment population of 176774 , as identified by Family Health Service register of September 2000. ${ }^{2}$

Optometrists initiated the referrals in all but one case, where the referral was direct from a general practitioner. The diagnosis of keratoconus was made by an ophthalmologist based on a history of progressive, irregular, myopic astigmatism and clinical assessment to identify signs consistent with keratoconus, as shown in Table $1{ }^{1,3}$

The ethnic origin of the patient was defined as white, Asian, or other via patient records or name in the few unstated cases. The ethnic breakdown of this population was identified as $82 \%$ white, $17 \%$ Asian, and $1 \%$ other by the Family Health Service register 2000. ${ }^{2}$ The proportion of the population other than white or Asian, being only $1 \%$, was considered negligible in this study. The age distributions of the two populations was also found from the Family Health Service register. ${ }^{2}$

Data were recorded concerning the patients' symptoms, sex, age of presentation, history of atopic disease, underlying disease, and family history as recorded in the patient records.

Statistical analysis was performed using $t$-test and confidence intervals $(\mathrm{CI})$, as applied to binomial data, for the incidence of keratoconus in Asians compared to white patients, female compared to male and the age of presentation. The incidence of atopic disease was compared in the two groups using $\chi^{2}$ tests of significance.

\section{Results}

In total, 74 cases of keratoconus were diagnosed over this 6 -year period. The patient records of all these patients were obtained. Of these patients, $29(39 \%)$ were white and $45(61 \%)$ were Asian. This equated to an incidence of keratoconus of 25 per 100000 (1 in 4000) (95\% CI 18-33) per year for Asians, compared with 3.33 per 100000 (1 in $30000)(95 \%$ CI $2.2-4.7)$ per year for white patients, presenting to hospital: a relative incidence of $7.5: 1$. The incidence was significantly higher in the Asian population compared to the white population $(P<0.001)$ (Figure 1).

Keratoconus presented in our study between the ages of 13 and 36 years. Within this age range, white

Table 1 Clinical signs of keratoconus

Protrusion of corneal apex (Munson's sign)

Corneal thinning

Fleischer's ring

Subepithelial scarring

Vertical stress lines (Vogt's striae)

Prominent corneal nerves

Scissoring reflex on retinoscopy

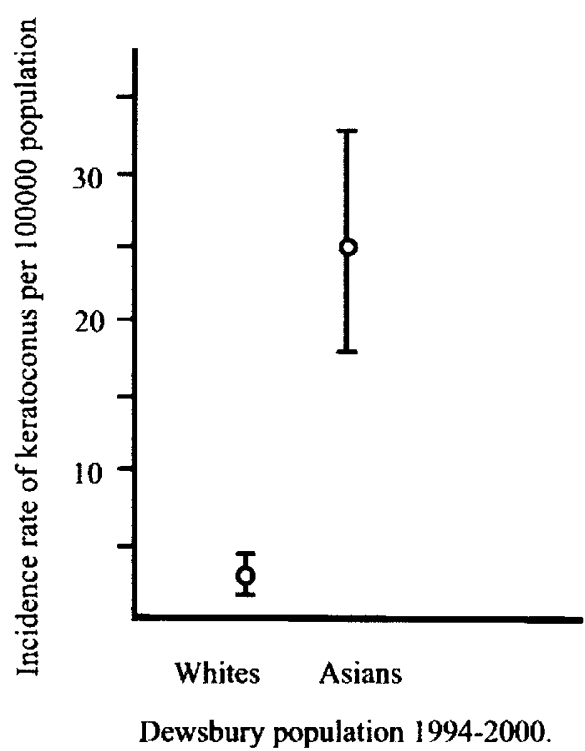

Figure 1 Incidence of keratoconus in white and Asian patients.

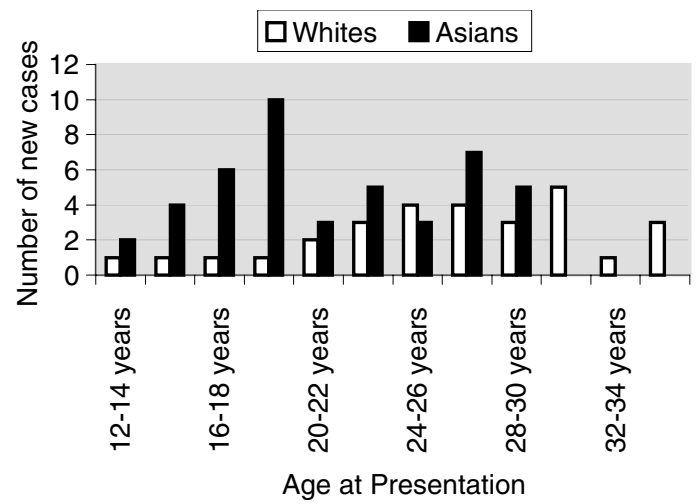

Figure 2 Age of presentation of keratoconus in Asian and white patients.

people accounted for $75 \%$ and Asians $25 \%$ of the population. ${ }^{2}$

Keratoconus was found to be significantly more common in males, $53(72 \%)$, than in female patients, 21 (28\%), $P<0.001$. There was no difference in the sex of patients presenting with keratoconus, white patients (21 (72\%) male, eight (28\%) female) compared to Asians (32 (71\%) male, 13 (29\%) female).

Asians presented significantly younger than white patients, with mean age of presentation for Asians of 21.5 years (95\% CI 19.4-23.6 years) and white patients 26.4 years (95\% CI 25.0-27.8), $(P<0.001)$ (Figure 2). Atopic disease was present in 15 of 29 (52\%) white patients and 10 of $45(22 \%)$ Asian patients, presenting with keratoconus. The incidence of atopy was significantly higher in white patients compared to Asian patients $(P<0.01)$ (Figure 3). Asthma was the most common form 


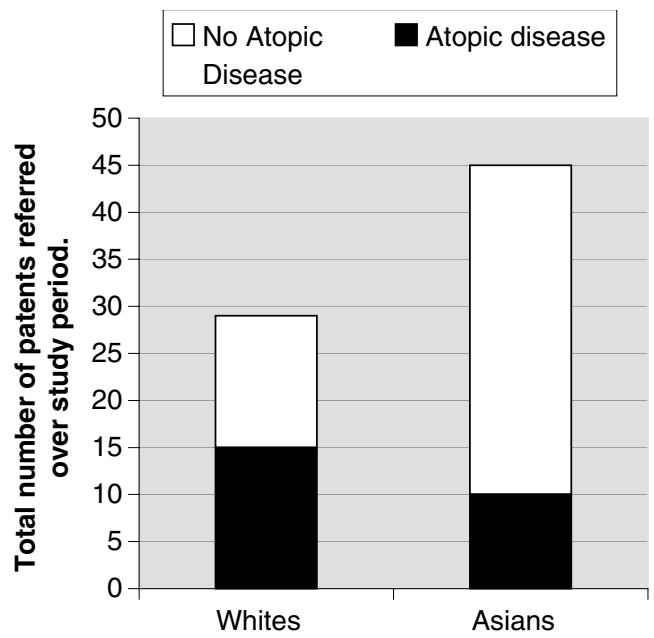

Figure 3 Incidence of atopy in white and Asian keratoconic patients.

of atopy occurring in $38 \%$ white patients compared to $18 \%$ Asian patients, eczema in $14 \%$ white patients compared to $7 \%$ Asian patients, and hay fever $20 \%$ white patients and 9\% Asian patients.

Initial treatment was not significantly different between the two groups. Of 29 white patients, 15 (52\%) and of 45 Asians, 20 (44\%) were prescribed glasses. Of 29 white patients, $12(41 \%)$ and of 45 Asians, 20 (44\%) required contact lens. Two white and five Asian patients were referred to the regional tertiary referral centre, Leeds General Infirmary, and had penetrating keratoplasty.

\section{Discussion}

In this study of patients from Dewbury, Asians were significantly more likely to present to hospital with keratoconus than white patients. This was highly suggestive of a greater incidence of the disease in this population. The incidence of the disease presenting to hospital was 25 per 100000 Asians per year, compared with 3.3 per 100000 white patients, a ratio of $7.5: 1$.

The population structure was $75 \%$ white and $25 \%$ Asian in the age range of presentation of keratoconus in this study. ${ }^{2}$ The greater incidence in Asians cannot, therefore, be accounted for by a tendency for Asian populations to be younger. The results showed a higher incidence in Asians than previously reported. Pearson et $a l^{4}$ found an incidence of keratoconus of 19.6 per 100000 in Asians and 4.5 per 100000 in white patients, a ratio of $4.4: 1$. An incidence of keratoconus of 2.2 and 2 per 100000 were found in studies of white people in Finland and Minnesota, respectively. ${ }^{5,6}$ Other estimates of incidence have varied from 1.4 to 230 per $100000 .^{7}$ The differences found in this study and that of Pearson et al showed that ethnic origin influenced incidence and was highly suggestive of a genetic factor being involved in the aetiology.

It was found that Asian patients presented at a statistically younger age than white patients, a mean age of 21.5 vs 26.4 years. This suggested that the Asian patients suffered a more severe form of the disease. Initial treatments were, however, comparable, except for an earlier trend towards more Asians being referred for consideration of penetrating keratoplasty (five of 45 compared with two of 29). Review of subsequent treatment may have revealed differences, with an assumed greater number of years of disease activity in those presenting younger. These findings were supported by those of Pearson et al, ${ }^{4}$ who showed Asians presented at a mean of 22.3 years compared to white people at 26.5 years $(P<0.0001)$. This study also revealed that Asians required corneal grafting at an earlier age and possibly had an increased speed of disease progression.

Atopy has been proposed as important in the aetiology of keratoconus and an association has been shown in many studies. ${ }^{1,3}$ One study in Florida found a prevalence of asthma of $0.4-1 \%$ in controls compared with $17.9 \%$ in keratoconus. ${ }^{8}$ A study of patients with vernal keratoconjunctivitis, in Turkey, found an incidence of keratoconus, as diagnosed by videokeratography, of $26.8 \% .^{9}$ A study in Dundee revealed an associated skin disorder in $34 \%$ of patients, hay fever in $36 \%$, and asthma in $21 \% .^{10}$

The incidence of atopic disease was found to be significantly higher in white patients compared to Asian patients with keratoconus. The incidence of atopy in the UK has been reported to be at least as high, if not higher in Asians than white people. A study in Leicester found an incidence of atopy of $38.4 \%$ in Asians vs $29.5 \%$ in white people $(P=0.01) .{ }^{11}$ The lower incidence of atopic disease in Asian keratoconic patients compared with white population gives support to the proposal of a different causation of disease in this population.

The aetiology of keratoconus is still unknown. Many factors both environmental and genetic have been suggested. The higher incidence of keratoconus in Asians compared with white population suggests that there is a genetic factor causing this higher than expected incidence. The vast majority of the Dewsbury Asian population originated from Northern Pakistan. This population has a tradition of consanguineous, especially first-cousin marriages. A study of 4738 mothers in Birmingham, including 956 of Pakistani origin, showed a prevalence of parental consanguinity of $69 \%$ in the Pakistani Muslims, 23\% in Muslims from other countries, and less than $1 \%$ in non-Muslims. ${ }^{12}$ In populations with a high prevalence of consanguinity, the incidence of 
genetic disease is higher than usually found. ${ }^{12}$ Several cases have been reported in the literature of keratoconus in children of consanguineous parents. ${ }^{13,14}$

In this study, only two Asian patients admitted to a family history of keratoconus. These relatives were not available for examination and so keratoconus could not be confirmed. The information on relatives was not complete and therefore some cases of positive family history may have been missed, an inherent problem with a retrospective study.

Evidence for the role of genetics includes twin studies. At least eight cases of keratoconus in both of homozygous twins have been reported. ${ }^{7,15}$ There are, however, some reports of discordance also. ${ }^{16}$ There have been many family studies suggesting a genetic cause. ${ }^{7}$ The pattern, however, does not appear clear. The majority of reported studies suggest an autosomal dominant mode of inheritance with variable expression. At least 74 incidences of families following this pattern have been reported. ${ }^{7}$ With this variable expression, it is probable that many other patients in these pedigrees have subclinical disease. It is hoped the increased use of videokeratography may reveal pedigrees suitable for biochemical and molecular study.

There is an association of keratoconus with trisomy 21, with a reported incidence of $5-10 \%$ in this population. ${ }^{14}$ A case of keratoconus in the daughter of a lady with a balanced translocation between the long arms of chromosomes 7 and 11, and pericentric inversion of 7 has suggested that a candidate gene for keratoconus may be located at or near this break point. ${ }^{17}$

The pathogenesis of keratoconus is unknown. It may be due to abnormalities in the corneal epithelium, the stromal keratocytes or an extracellular matrix component. There may be a structural abnormality of collagen or the products involved in its regulation. High rates of mitral valve prolapse have been associated with keratoconus suggesting a connective tissue cause. ${ }^{14}$

It is probable that keratoconus is a spectrum rather than a single identifiable disease; the end result of several different pathological processes. If this is correct, the progress in identifying the aetiology will be more rapid if studies are confined to specific populations and forms of keratoconus.

This study has several weaknesses. Firstly, as it is retrospective and relies on accurate full clinical notes. The diagnosis of keratoconus was not subject to strict predetermined criteria. The estimates of incidence rely on family health service records. These, however, are likely to underestimate rather than overestimate the Asian population, as these patients are traditionally less likely to register with a general practitioner.

This study looked at only those patients who presented to the hospital with usually symptomatic keratoconus.
The higher incidence in Asians may represent a more severe form of the disease rather than an actually higher incidence. The number of people with keratoconus treated in the community by optometrists is unknown. The study may be criticized for ignoring all non-white non-Asian patients. This, however, was felt to be a small enough number to not cause any statistical difference. In further studies, it would be advisable to define ethnic origin more carefully. The province that the family originates from and their religion should be ascertained.

This study adds significant weight to the theory of keratoconus as a condition with an underlying genetic cause or susceptibility. Further studies are required to look at the Asian populations, using videokeratography along with clinical examination to identify families where genetic linkage analysis can be used to identify underlying genetic causation.

\section{Acknowledgements}

Acknowledgements to Dr Rory O'Conor, Consultant Epidemiologist, Dewsbury District Hospital, for his assistance in the statistical analysis.

\section{References}

1 Bawazeer AM, Hodge WG, Lorimer B. Atopy and keratoconus: a multivariate analysis. $\mathrm{Br} J$ Ophthalmol 2000; 84: 834-836.

2 Family Health Service Register. Calderdale and Kirklees Health Authority, Patient Index Data Base. St Luke's House, Huddersfield, September 2000.

3 Kenyon RK, Hersh PS, Starck T, Fogle JA. Corneal dysgenesis, dystrophies and degenerations. In: Tasman W, Jaeger E (eds). Duane's Ophthalmology. Lippincott Williams \& Wilkins: Baltimore, 1997; 4(16): 1-53.

4 Pearson AR, Soneji B, Sarvananthan N, Sandford-Smith JH. Does ethnic origin influence the incidence or severity of keratoconus? Eye 2000; 14(Part 4): 625-628.

5 Ihalainen A. Clinical and epidemiological features of keratoconus: genetic and external factors in the pathogenesis of the disease. Acta Ophthalmol 1986; 178(Suppl): 5-64.

6 Kennedy RH, Bourne WM, Dyer JA. A 48 year clinical and epidemiological study of keratoconus. Am J Ophthalmol 1986; 101: 267-273.

7 Rabinowitz YS. Keratoconus - major review. Surv Ophthalmol 1998; 42(4): 297-318.

8 Gasset AR, Hinson WA, Frias JL. Keratoconus and atopic diseases. Ann Ophthalmol 1978; 10(8): 991-994.

9 Totan Y, Hepsen IF, Cekic O, Gunduz A, Aydin E. Incidence of keratoconus in subjects with vernal keratoconjunctivitis: a videokeratograhpic study. Ophthalmology 2001; 108: 824-827.

10 Weed KH, McGhee CNJ. Referral patterns, treatment management and visual outcome in keratoconus. Eye 1998; 12: 663-668. 
11 Carey OJ, Cookson JB, Britton J, Tatersfield AE. The effect of lifestyle on wheeze, atopy, and bronchial hyperreactivity in Asian and white children. Am J Respir Crit Care Med 1996; 1549(1): 537-540.

12 Bundey S, Alam H, Kaur A, Mir S, Lancashire RJ. Race, consanguinity and social features in Birmingham babies: a basis for prospective study. J Epidemiol Community Health 1990; 44(2): 130-135.

13 Duke-Elder S, Leigh AG. Keratoconus. In: Duke-Elder S (ed). System of Ophthalmology, Vol 8, No. 2. Mosby: St Louis, MO, 1965, pp 964-976.
14 Jacobs DS, Dohlman CH. Is keratoconus genetic? Int Ophthalmol Clin 1993; 33(2): 249-260.

15 Bechara SJ, George O, Waring III, Insler MS. Keratoconus in two pairs of identical twins. Cornea 1996; 15(1): 90-93.

16 McMahon TT, Shin JA, Newlin A, Edrington TB, Sugar J, Zadnik K. Discordance for keratoconus in two pairs of monozygotic twins. Cornea 1999; 18(4): 444-451.

17 Morrison DA, Rosser EM, Claoue C. Keratoconus associated with a chromosome 7, 11 translocation. Eye 2001; 15(4): 556-557. 